\title{
Vazōes relativas dos rios Negro e Solimões através das concentrações de ${ }^{18} \mathrm{O}^{(1)}$
}

\author{
E. Matsui (*) \\ E. SALATi $(*)$ \\ W. L. F. BRINKMANN (**) \\ I. FRIEDMAN (***)
}

\begin{abstract}
SINOPSE
Foram encontradas concentrações isotopicas di. ferentes nas águas do Rio Negro e do Rio Solimíes. Partindo daquela verificação preliminar, foi feita a amostragem sistemática de águas daqueles dois rios e do rio Amazonas, procurando-se determinar abaixo da confluência dos mesmos, o local onde o rio Amazonas apresenta uma estrutura horizontal e vertical homogênea traduzindo perfeita mistura das águas dos rios formadores. Os dados obtidos permitem também o cálculo, com razoável precisão, da vasão relativa de ambos os rios para alguns meses. Verifi. cou-se que a $120 \mathrm{Km}$ da confluência do Negro e o Solimões, ainda não havia mistura perfeita e que abaixo daquele ponto, a confluência do Madeira introduz perturbações na mistura

O grau de mistura em diferentes pontos apresenta oscilações em amostras colhidas em diferentes meses. Os dados obtidos pela medida da concentração isotopica concordam sensivelmente com os resultados das medidas diretas efetuadas por Oltman et al (1963).
\end{abstract}

\section{INTRODUÇÃO}

A composição isotópica das águas de precipitação depende de um grande número de fatores os quais estão intimamente ligados com a dinâmica do ciclo hidrológico (Friedman et al., 1564; Salati et al., 1971).

Os fatores determinantes da composição isotópica da água de precipitação são:

a. A origem do vapor de água que dará forma. ção às precipitações;

b. Temperatura e mecanismo de condensação; c. Trocas isotópicas entre a gota de chuva com o vapor d'água do meio ambiente. (Friedman, 1962).

d. Evaporação da gota de chuva durante a precipitação (Woodcock \& Friedman, 1963; Dansgaard, 1964) .

Os fatores citados acima realmente integram processos mais detalhados nos mecanismos de condensação e evaporação, porém já dão uma idéia que pode haver uma grande variação das concentrações isotópicas nas águas de precipitação (Eriksson, 1965; (Craig \& Gordon, 1965; Gat et al., 1972). Estas variações têm sido estudadas em todo globo terrestre (Dansgaard, 1964) sendo que alguns poucos dados foram obtidos na Amazônia e publicados pela International Atomic Energy Agency (1971).

Em determinações preliminares (Salati et a!., 1970) verificou-se que as concentraçōes isotópicas das águas dos rios Negro e Solimões eram diferentes; estas diferenças podem ser facilmente explicadas tendo-se em vista as regiões de recarga daquelas bacias hidrográficas. O rio Negro (Fig. 1) tem alguns formadores vindos do hemisfério Norte e o rio Solimões tem sua alimentação em rios provenientes do hemisfério Sul. Do hemisfério Norte ainda sofre a influência das águas de degelo das neves Andinas (Salati et alii, 1973; Soares, 1959). Com base nestes dados preliminares iniciou-se uma amostragem sistemática de águas daqueles rios incluindo também medidas do rio Amazonas abaixo da confluência dos rios Negro e Solimões

(1) - Trabalho realizado com o auxílio da Comissão Nacional de Energia Nuclear, Fundação de Amparo à Pesquisa do Estado de São Paulo e International Atomic Energy Agency. A coordenação dos trabalhos foi feita pelo Departamento de Pesquisa Científica e Tecnológica da CNEN.

(*) - Centro de Energia Nuclear na Agricultura, USP - CNEN

(**) - Instituto Nacional de Pesquisas da Amazônia.

${ }^{(* * *)}-$ U. S. Geological Survey. 


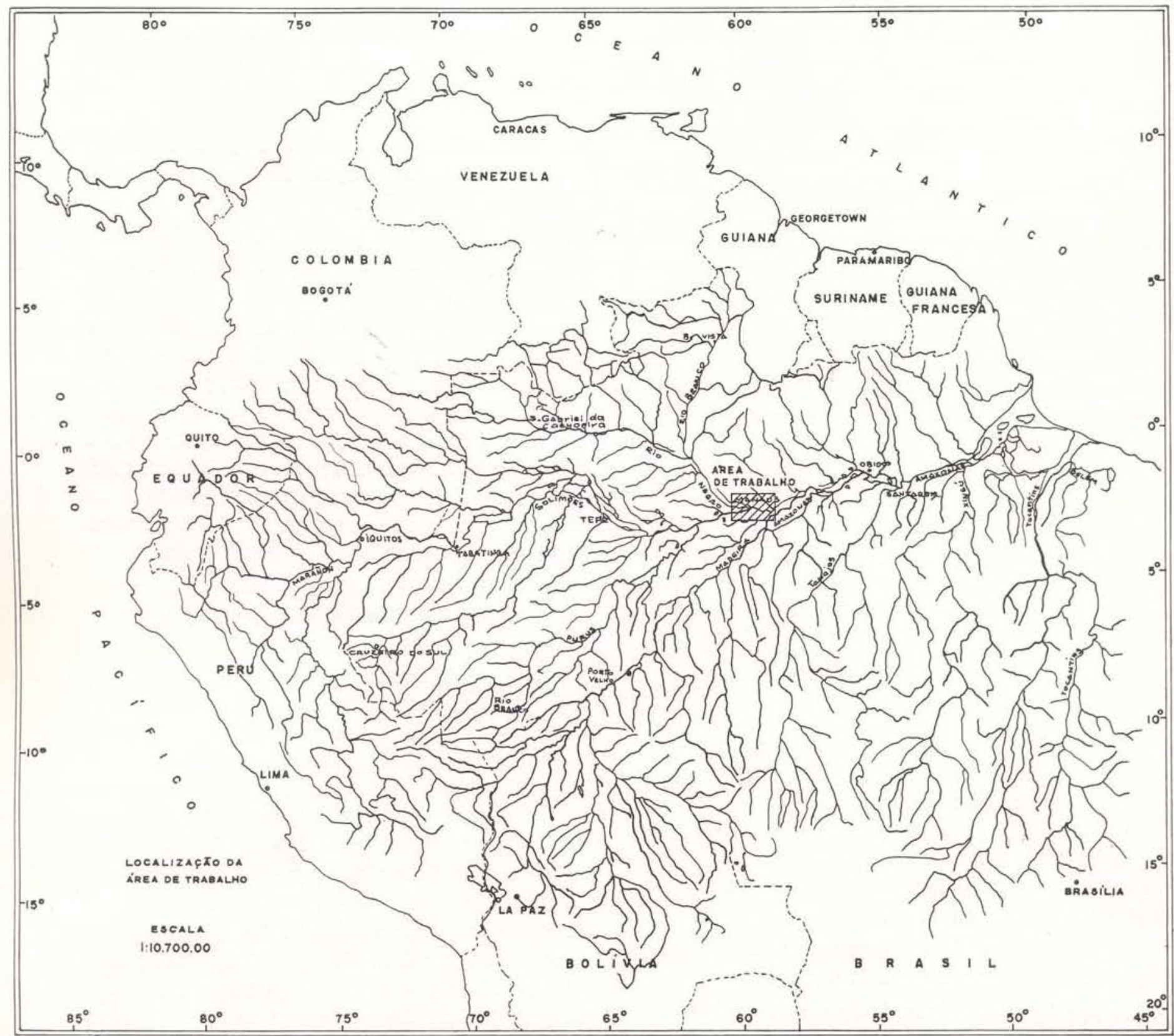

Fig. 1 - HIDROLOGIA ISOTÓPICA DA AMAZÔNIA - Vazóes relativas dos ríos Negro e Solimões através das concentrações de $18_{0}$ 
A fim de se estimar as vazões relativas dos rios Negro e Solimōes procurou-se determinar abaixo da confluência dos mesmos, o local oncie o rio Amazonas apresenta uma estrutura ver tical e horizontal homogênea, isto é, onde as águas dos rios formadores estão já perfeitamente misturadas. Encontrou-se alguma dificuldade em se determinar este local entre a foz do rio Negro e a do Madeira onde as águas do rio Solimões e Negro formassem uma mistura homogênea. Estes problemas são discutidos no parágrafo 3 .

Durante um ano foram feitas observações sistemáticas no rio Negro perto da cidade de Manaus; no rio Solimões nas proximidades de Manaus e no rio Amazonas, em S. José do Amatarí mais ou menos $120 \mathrm{~km}$ abaixo do encontro des águas. Com os dados obtidos foi possivel estabelecer as variações isotópicas dos rios e também estimar com um nível razoável de precisão as vazões relativas dos rios Negro e Solimões para alguns meses. Em se tratando de uma região onde os dados hidrológicos são escassos (apenas uma medida de precisão foi feita da vasão desses rios por Oltman e outros, (1964), o método apresenta-se promissor e pode ser aplicado a outros locais da Bacia Amazôni$\mathrm{ca}$, nos quais medidas de vazões não foram estudadas ainda. Por outro lado, basta que um valor absoluto da vazão seja conhecido para cue se possa calcular os outros dois.

\section{MÉTODO DE TRABALHO}

\section{LOCAIS DE AMOSTRAGENS :}

No rio Negro as amostram foram coletadas ao longo de uma linha transversal que passa pela ilha do Marapatá, $5 \mathrm{~km}$ acima da foz sendo as amostras coletadas a diversas profundidades nas margens direita, esquerda e no meio do rio, de acordo com os esquemas indicados nas figuras 4 a 10.

No rio Solimões as amostras foram coletadas ao longo de uma linha transversal $30 \mathrm{~km}$ acima da foz, perto da llha de Jacurutu de acordo com os esquemas indicados nas figuras 4 a 10 ."

As amostras sistemáticas do rio Amazonas foram feitas ao longo de um corte transversal a aproximadamente $120 \mathrm{~km}$ abaixo do encontro dos rios Negro e Solimões nas proximidades de S. José do Amatarí e os pontos de coleta estão indicados esquematicamente nas figuras de 4 a 10.

TÉCNICA DE MEDIDA:

As medidas das concentrações de ${ }^{18} 0$ foram feitas pelos métodos convencionais de espectrometria de massa McKinney, et al., 1950; Epstein \& Mayeda, 1953. Para medida de ${ }^{18} 0$ utilizouse um Espectrômetro de Massa da Varian-Mat modelo $\mathrm{CH}-4$ especialmente adaptado, nos trabalhos de rotina de nossos laboratórios o desvio padrão é para $\delta 18= \pm 0,17^{\circ} \%$ oo.

Nestas pesquisas no entanto procurou-se aumentar a precisão relativa das medidas e para isso tomou-se como referência de trabalho uma das amostras de água, geralmente a água do próprio rio Amazonas e determinou-se as variações das composições isotópicas das outras amostras em relação a ela. Conseguiu-se, por esta técnica, para oxigênio uma precisão da ordem de $\delta 18= \pm 0,05^{\circ} \%$, e pode-se atingir uma precisão da ordem de $\pm 0,02 \%$.

ESTIMATIVAS DAS VAZÕES RELATIVAS POR DILUIÇÃO ISOTÓPICA

Se as águas de dois rios possuem concentrações isotópicas, $\mathrm{D}$ ou ${ }^{18} 0$, diferentes, pode-se determinar a vazão relativa de um rio em relação ao outro, desde que o rio formado por esses dois percorra uma distância suficiente para que haja uma homogeneização da mistura das águas. antes que receba outro afluente.

Sejam os afluentes $r_{1}$ e $r_{2}$ indicados na $\mathrm{fi}$. gura 4 , os formadores do rio $r_{3}$.

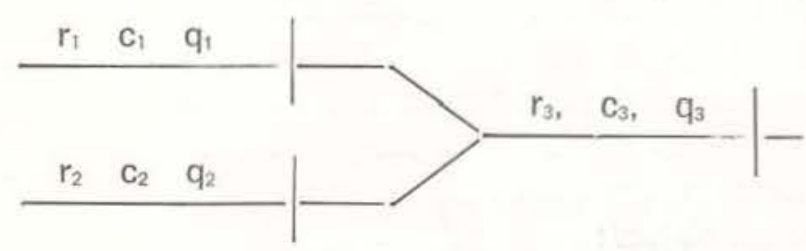

Sejam $\mathrm{c}_{1}$ e $\mathrm{c}_{2}$ as concentrações de ${ }^{18} 0$ nas aguas dos rios $r_{1}$ e $r_{2}$, respectivamente, e $c_{3}$ a concentração da mistura no rio $r_{3}, e q_{1}, q_{2}$ e $q_{3}$ as vazões dos rios $r_{1}, r_{2}$ e $r_{3}$, respectivamente. A concentração absoluta de ${ }^{18} 0$ e a soma das concentrações absolutas de ${ }^{16} 0,{ }^{17} 0$ e ${ }^{18} 0$. 
O balanço isotópico para ${ }^{18} 0$ será :

$$
\mathrm{q}_{3} \mathrm{C}_{3}=\mathrm{q}_{1} \mathrm{c}_{1}+\mathrm{q}_{2} \mathrm{C}_{2} \ldots \ldots \ldots \ldots \ldots \ldots
$$

e o balanço da quantidade de água será :

$$
q_{3}=q_{1}+q_{2}
$$

de (2) tiramos que :

$$
1=\frac{q_{1}}{q_{3}}+\frac{q_{2}}{q_{3}}
$$

Se fizermos :

$$
\frac{q_{1}}{q_{3}}=x \text {, então } \frac{q_{2}}{q_{3}}=1-x \ldots \ldots
$$

Pela equação (1) tem-se :

$$
c_{3}=\frac{q_{1}}{q_{3}} c_{1}+\frac{q_{2}}{q_{3}} c_{2} \ldots \ldots \ldots \ldots
$$

substituindo (3) em (4) :

$$
\begin{aligned}
& c_{3}=x \cdot c_{1}+(1-x) c_{2} \\
& c_{3}=x c_{1}+c_{2}-x c_{2} \\
& x=\frac{c_{3}-c_{2}}{c_{1}-c_{2}} \ldots \ldots
\end{aligned}
$$

e a equação que dá a fração contribuída pelo rio $r_{1}$.

Pela expressão (5) pode-se ver que o valor de $x$ depende somente das diferenças das concentrações de ${ }^{18} 0$ e não dos valores absolutos dessas concentrações e o método de análise utilizado permitiu obter medidas das diferenças das concentrações isotópicas com precisão meIhor do que $0,05 \%$. Como no processo de medida utilizado o que se determina é o desvio da relação ${ }^{18} 0 /{ }^{16} 0$ entre as amostras, devemos adaptar a expressão (5) às medidas realizadas, assim, sejam :

$$
A=\frac{{ }^{18} 0}{{ }^{16} 0} \text { da amostra }
$$

${ }^{\mathrm{p}} \mathrm{p}=\frac{{ }^{18} 0}{{ }^{16} 0}$ de um padrão cujo valor é conhecido entẵo :

$\delta A=\frac{{ }^{r} A-r P}{r p}$, daí ${ }^{r} A=r P(\delta A+1) \ldots$.

As concentrações naturais dos isótopos de oxigênio estão aproximadamente na seguinte proporção: ${ }^{16} 0:{ }^{17} 0:{ }^{18} 0=997600: 400: 2000$.

Daqui se tem :

$\frac{{ }^{18} 0}{{ }^{16} 0+{ }^{17} 0+{ }^{18} 0}=\frac{{ }^{18} 0}{{ }^{16} 0}(7)$ com boa aproximação.

F'elas relações (6) e (7), a equação (5) torna-se :

$$
x=\frac{\delta r_{3}-\delta r_{2}}{\delta r_{1}-\delta r_{2}}
$$

Sendo a vazão relativa calculada pela expressão (8) o erro de $x$, em função dos erros de $\delta r_{1}, \delta r_{2}$ e $\delta r_{3}$ é estimado como segue :

Para Deutério :

$$
\begin{aligned}
& \Delta \text { Rio Amazonas }=\Delta \text { Rio Solimões }= \\
& \Delta \text { Rio Negro }=1 \%
\end{aligned}
$$

Para Oxigênio:

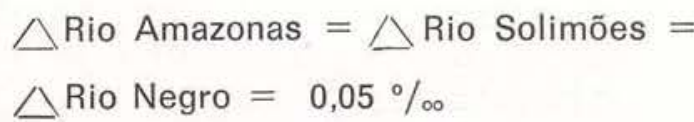

DADOS OBTIDOS E DISCUSSÕES :

VALORES MÉDIOS DAS CONCENTRAÇÕES ISOTÓPICAS

Os valores obtidos para as concentrações de ${ }^{18} 0$ estão indicados no Quadro I. As amostras foram coletadas por volta do dia 15 de cada mês. Pode-se verificar pela Figura 2, que segundo o regime dos rios as concentrações isotópicas são bem diferentes, podendo-se aplicar o método descrito no parágrafo 2 e em outros meses as diferenças são tão pequenas que o método não pode ser aplicado com bons resultados práticos. 


\begin{tabular}{l|c|c|c}
\hline Data & $\begin{array}{c}\text { Rio } \\
\text { Negro } \\
{ }^{18} 0\end{array}$ & $\begin{array}{c}\text { Rio } \\
\text { Solimões } \\
{ }_{18} 0\end{array}$ & $\begin{array}{c}\text { Rio } \\
\text { Amazonas } \\
\text { 180 }\end{array}$ \\
\hline $09 / 71$ & $-4,8$ & $-5,6$ & - \\
$10 / 71$ & $-4,1$ & $-5,3$ & - \\
$11 / 71$ & $-4,5$ & $-6,2$ & - \\
$12 / 71$ & $-4,6$ & $-6,2$ & - \\
$01 / 72$ & $-4,6$ & $-6,1$ & - \\
$02 / 72$ & $-4,5$ & $-6,5$ & - \\
$03 / 72$ & $-4,5$ & $-6,7$ & - \\
$04 / 72$ & $-5,0$ & $-7,6$ & $-6,9$ \\
$05 / 72$ & $-5,7$ & $-8,0$ & $-7,1$ \\
$06 / 72$ & $-7,9$ & $-8,1$ & $-7,9$ \\
$07 / 72$ & $-6,6$ & $-7,6$ & $-7,2$ \\
$08 / 72$ & $-5,9$ & $-7,0$ & $-6,6$ \\
$09 / 72$ & $-4,6$ & $-5,7$ & $-5,2$ \\
$01 / 73$ & $-4,0$ & $-5,8$ & $-5,4$ \\
\hline
\end{tabular}

QUADRO 1 - Dados médios de $\delta^{i 8}$ dos rios $\mathrm{Ne}$ gro, Solimões e Amazonas nos locais indicados na Figura 4. Erro da medida: $\pm 0,2^{\circ} / 00$.

\section{HOMOGENEIDADE DA MISTURA DAS ÁGUAS} DOS RIOS NEGRO E SOLIMÕES

Como indicou-se no parágrafo 2 , para que - cálculo das vazões relativas pelo método de diluição isotópica possa ser utilizado, é necessário que haja uma completa mistura das águas dos rios formadores. As primerias observaçōes para se determinar o local em que as águas dos rios Negro e Solimões estivessem misturadas, foram feitas sobrevoando-se a região de encontro das águas e observando-se a cor das mesmas que são perfeitamente distinguíveis. As águas do rio Negro são escuras justificando o nome que tem e as águas do Solimões apresen. tam-se geralmente amarelada-barrenta; a primeira indicação foi que aparentemente a $40 \mathrm{~km}$ da foz os rios estavam misturados, distância esta que depende da época do ano. Posteriormente foram feitas amostragens para análise isotópica em alguns cortes transversais do rio
Amazonas abaixo de Manaus, foi possivel verificar que a completa mistura não havia se realizado nem $120 \mathrm{~km}$ abaixo. Os dados da fig. 3 dão uma idéia da dimensão do problema.

A dificuldade da mistura lateral de rios já foi salientada por Krouse \& Mekay (1971) que utilizando o método de diluição isotópica das concentrações de ${ }^{18} 0$ estudaram o problema da mistura das águas dos rios Liand-Mackenzie no Canadá e observaram que a distância para uma completa homogeneização foi da ordem de $300 \mathrm{~km}$.

Em vista dos dados obtidos tomou-se como local para amostragem sistemática no Amazonas a cidade de S. José do Amatarí aproximadamente a $120 \mathrm{~km}$ da foz do Negro com o Solimões porque logo abaixo está a foz do rio Macieira o qual pelo volume de água que possue traz novas perturbações na mistura.

\section{CÁLCULO DA VAZÃo RELATIVA}

Os valores das concentrações isotópicas para estimativa da vazão estão indicados para cada mês nas figuras 4 a 10 , sendo que o erro padrão dos valores indicados foi da ordem de $\mathrm{U}, 05^{\circ} \%$ oo. Como pode-se ver pelos valores apresentados nos cortes transversais das figuras 4 a 10 as águas tanto do rio Negro como do Solimões eram perfeitamente homogêneas. Em 3. José do Amatarí, no entanto, a mistura lateral não estava completa, variando o grau de homogeneidade de acordo com o mes. Para cálculo da vazão, tomamos uma média dos valores obtidos em diversos pontos dos rios estudados e os vasos calculados com esses dados e com auxílio da expressão 8 , estão no quadro 2 .

Nos meses estudados observamos que a contribuição do Negro foi menor em janeiro com valor de $(20 \pm 5) \%$ e a maior contribuição ocorreu no mês de julho com valor de $(43 \pm 9) \%$.

Comparando os dados obtidos pelo método de diluição isotópica com os dados de medidas diretas de vazão realizadas por Oltman et al (1963) e admitindo-se que as vazões relativas foram semelhantes no ano estudado (1963) e em 1972, observamos existir uma boa concordância nos resultados, como pode-se ver pelo Quadro III. 


\begin{tabular}{|c|c|c|c|c|c|c|c|}
\hline \multirow{2}{*}{ D a $t$ a } & \multicolumn{4}{|c|}{$18\left( \pm 0,05^{\circ} / 00\right)$} & \multirow{2}{*}{$\begin{array}{c}\text { Vazão } \\
\% \\
\text { Rio Negro }\end{array}$} & \multirow{2}{*}{\multicolumn{2}{|c|}{$\begin{array}{c}\text { Vazão } \\
\% \\
\text { Rio Solimões }\end{array}$}} \\
\hline & Rio Negro & Rio & Solimốes & Rio Amazonas & & & \\
\hline Abril/ 72 & $-5,02$ & & $-7,61$ & $-6,91$ & $38 \pm 4$ & & $62 \pm 4$ \\
\hline Maio/72 & $-5,68$ & & $-8,00$ & $-7,07$ & $33 \pm 4$ & & $67 \pm 4$ \\
\hline Junho/72 & $-7,90$ & & $-8,05$ & $-7,94$ & - & & - \\
\hline Julho/72 & $-6,57$ & & $-7,64$ & $-7,18$ & $43 \pm 9$ & & $57 \pm 9$ \\
\hline Agosto/72 & $-5,90$ & & $-7,03$ & $-6,55$ & $42 \pm 9$ & & $58 \pm 9$ \\
\hline Setembro/72 & $-4,56$ & & $-5,68$ & $-5,22$ & $41 \pm 9$ & & $59 \pm 9$ \\
\hline Janeiro/73 & $-3,95$ & & $-5,80$ & $-5,43$ & $20 \pm 5$ & & $80 \pm 5$ \\
\hline
\end{tabular}

QUADRO II - Concentração de ${ }^{18} 0$ nos rios Negro e Solimōes nas proximidades de Manaus e no rio Amazonas em Amatarí. Vazões relativas dos rios Nezro e Solimões.

\begin{tabular}{|c|c|c|c|c|}
\hline Rio & $\begin{array}{l}\text { Oltman (1964) } \\
\text { Vazão (m } 3 / 5)\end{array}$ & $\begin{array}{c}\text { Oltman (1964) } \\
\%\end{array}$ & $\begin{array}{c}\text { Gessner (1959) } \\
\%\end{array}$ & $\begin{array}{c}\text { Este Trabalho } \\
\%\end{array}$ \\
\hline $\begin{array}{l}\text { NEGRO } \\
\text { (Manaus) }\end{array}$ & 64.967 & 38,4 & 40,0 & $43 \pm 9$ \\
\hline $\begin{array}{l}\text { SOLIMOES } \\
\text { (perto de Manaus) }\end{array}$ & 104.402 & 61,6 & 60,0 & $57 \pm 9$ \\
\hline $\begin{array}{l}\text { AMAZONAS } \\
\text { (S. José do Amatarí) }\end{array}$ & 169.369 & & & \\
\hline
\end{tabular}

QUADRO III - Vazões absolutas dos rios Negro, Solimões e Amazonas medidas por Oltman et al (1964) em julho de 1963 e vazões relativas medidas por Gessner (1959) em maio de 1959 e por diluição isotópica (julho de 1972).

\section{SUMMARY}

Isotopic concentration was detected in different spots of Rio Negro, Rio Solimões and Rio Amazonas at regular intervals, for 12 consecutive months. Values as found in Rio Negro waters were significantly different. Difference between isotopic concen. tration found in Rio Negro and Rio Solimões was considered as useful information for identifying the spot where Rio Amazonas, formed by the confluence of Negro and Solimōes, presents a homogenous vertical and horizontal structure after complete mixing of both rivers. A perfect mixture was not found up to $120 \mathrm{~km}$ below the confluence. Systematic sampling was found to be a method for the determi. nation of relative outflow of both rivers. Data as obtained are close to the results of direct measurements performed by Oltman et al (1963).

\section{BIBLIOGRAFIA CITADA}

Craig, H. \& Gordon, L. I.

1965 - Deuterium and Oxygen-18 variations in the Ocean and the Marine Atmosphere. In : Tongiorgi, E. ed. - Stable isotopes in Oceanographic studies and paleotemperatures. Pisa, Lab. Div. Geologia Nucleare. p. 9-130.

DANSGAARD, W.

1964 - Stable isotopes in precipitation. Tellus, 16: 436-468.

EPSTEIN, S. \& MAYEDA, T.

1953 - Variation of 180 content of waters from natural sources. Geochim. cosmochim. Acta, London 4: 213-224.

ERIKSSON, E.

1965 - Deuterium and Oxygen-18 in precipitation and other natural waters. Some theoretical considerations. Tellus, $16: 498-512$. 
FrIEDMAN, I.

1962 - Water-Vapor exchange between a water droplet and its environment. J. geophys Res., Baltimore, $67:$ 2761-2766.

FrIEDMAN, I. ET ALII

1964 - The Variation of the deutarium content of natural waters in the Hydrologic cycle. Rev. Geophys., 2: 177-223.

Gat, J. \& Dansganad, W.

1972 - Stable isotope survey of the fresh water occurrences in Israel and the Northern Jordan hift Valley. J. Hydrol., 16: 177-211.

GESSNER, F.

1959 - Hydrobotanik II. Berlin, Deutscher Verlag der Wissenschaften.

INTERNATIONAL ATOMIC ENERGY AGENCY

1971 - Environmental isotope data $n^{\circ} 3$ : World survey of isotope concentration in precipitation (19661967). Tech. Rep. Ser., 129.

Krouse, H. R. \& Mackay, J. R.

1971 - Application of $\mathrm{H}_{2}{ }^{18} 0 / \mathrm{H}_{2} \quad{ }^{10} 0$ abundances to the problem of lateral Mixing in the Liard Mackenzie river system. Can. J. Earth Sci.. 8: 1107-1109.
MCKINNEY, C. R. ET ALII

1950 - Improvements in Mass Spectrometers for the measurement of small differences in Isotope abundance ratios. Rev. scient. Instrum., New York, 21: 724-730.

OLTMAN, R, E. ET ALII

1964 - Amazon River investigations reconaissance measurements of July 1963. Circ. U.S. geol. Surv., 486.

SALATI, E. ET ALII

1970 - Concentraçâo de ${ }^{180}$ e $\mathrm{D}$ em amostras de água de rios do Brasil. (inédito).

1971 - Estudo preliminar das concentraçōes de ${ }^{180}$ e $D$ em águas do Nordeste Brasileiro.

1973 - Concentrações de ${ }^{18} 0$ e D em águas fluviais da Amazônia. CENA (no prelo.)

SOARES, L. C.

1959 - Hidrografia, a grande bacia amazônica. In : Brasil. Conselho Nacional de Geografia Geografia do Brasil, Grande Região Norte. Rio de Janeiro, IBGE. p. 128-194.

Woodcock, A. H. \& Friedman, I.

1963 - The Deuterium content of Raindrops, J. geophys Res., Baltimore, 68: 4477-4483. 


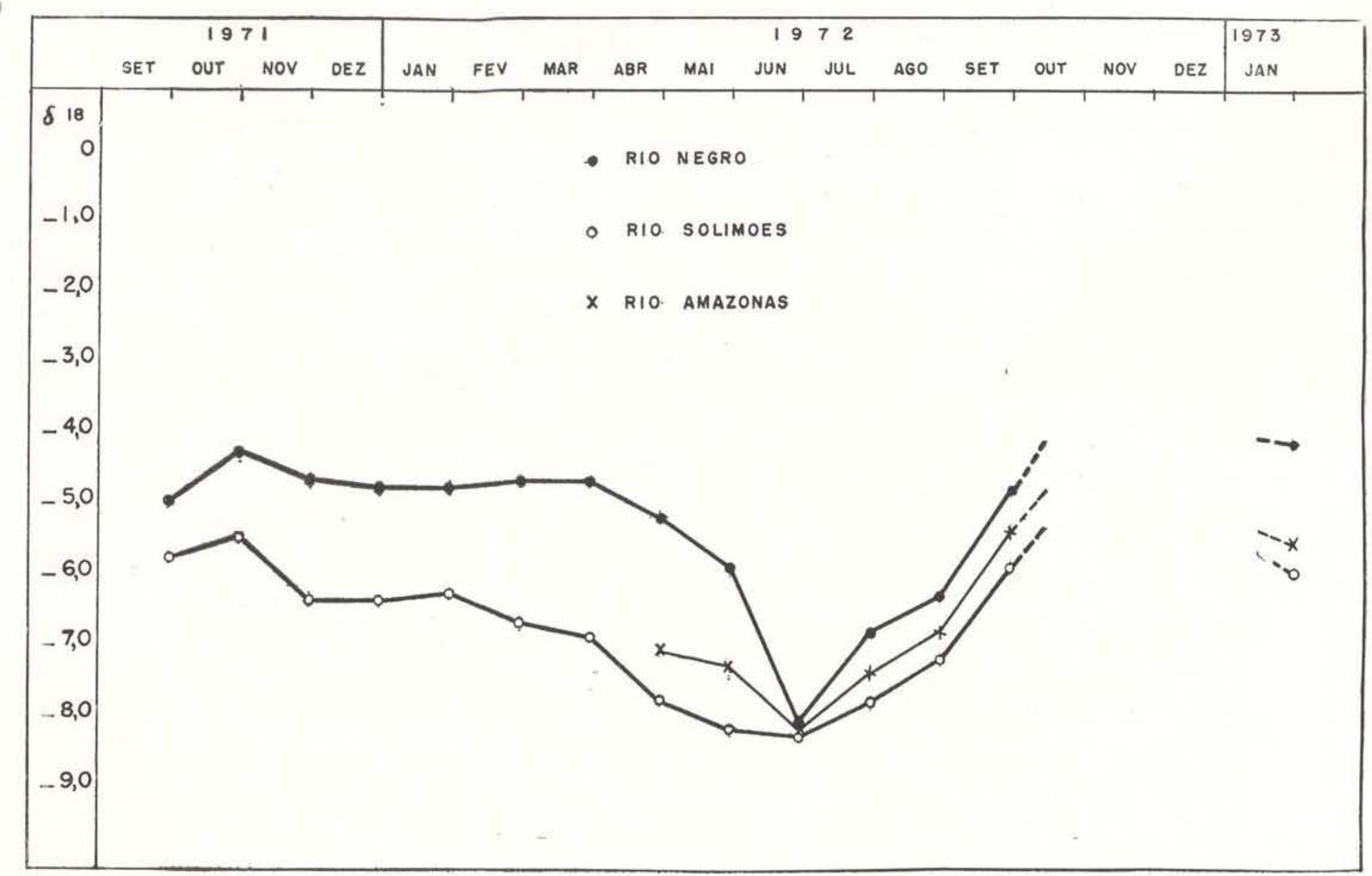

Fig. 2 - Variações de $\delta 18$ nos rios Negro (Manaus), Solimões (próximo de Manaus) e Amazonas (S. José do Amatari) 


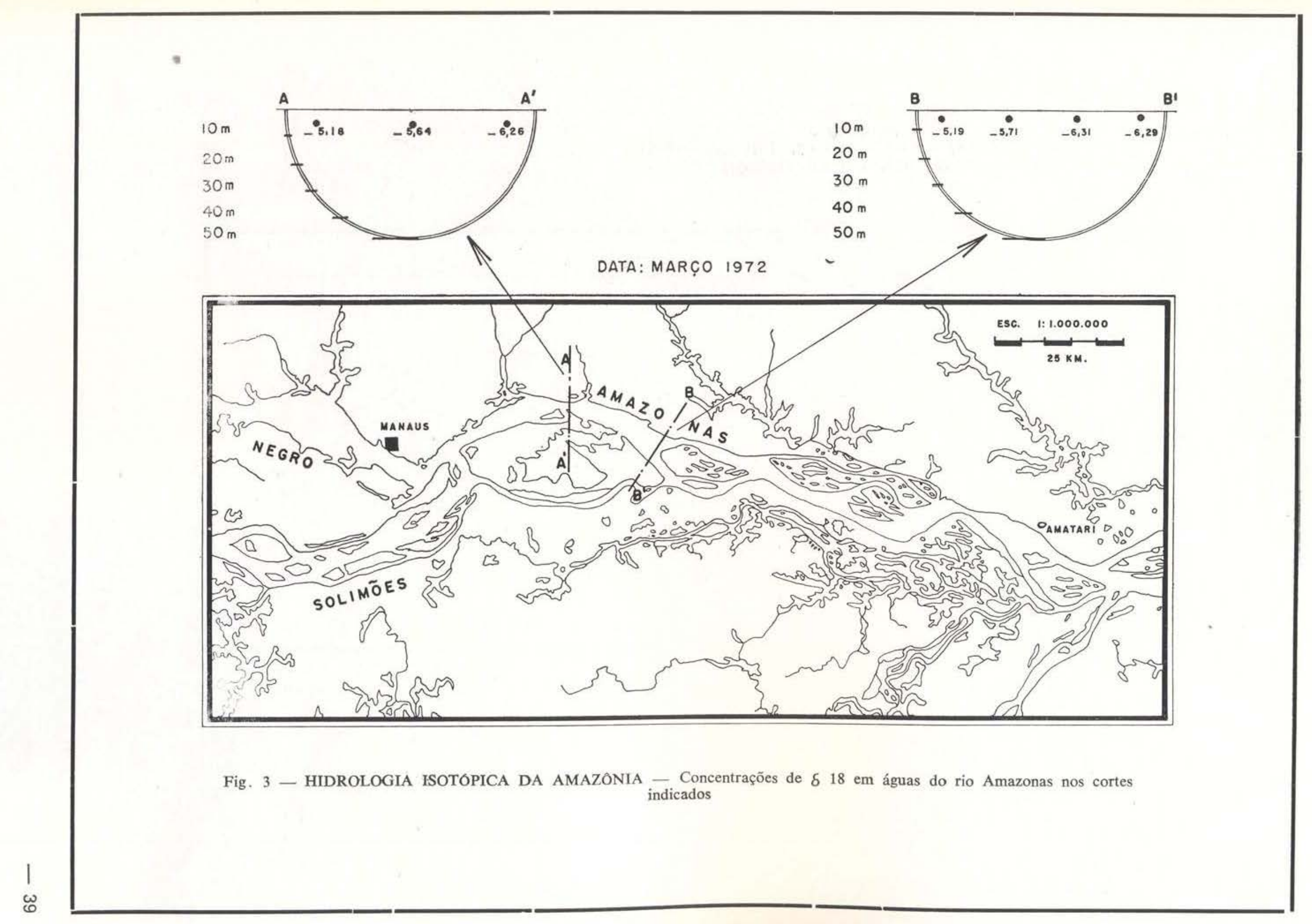




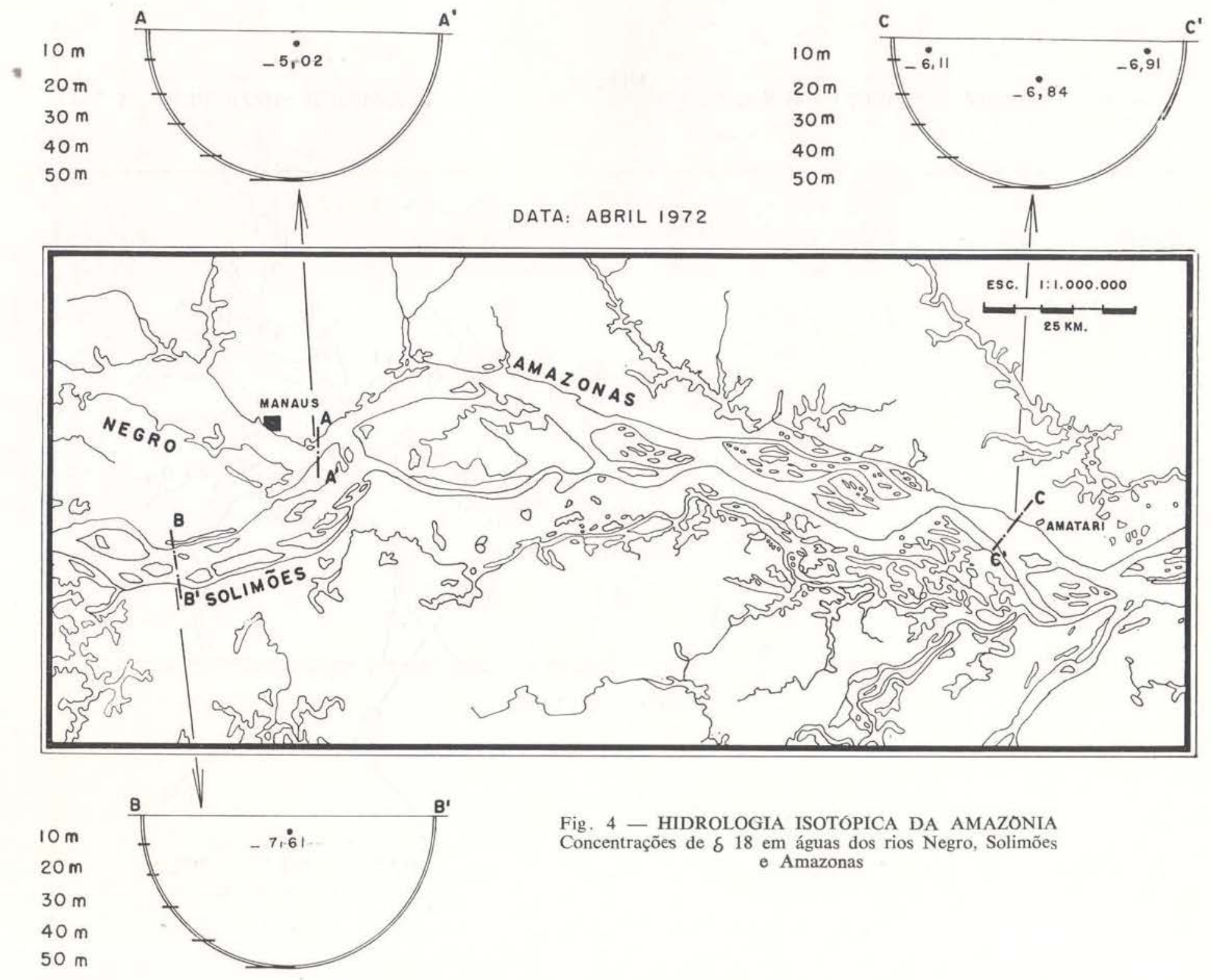




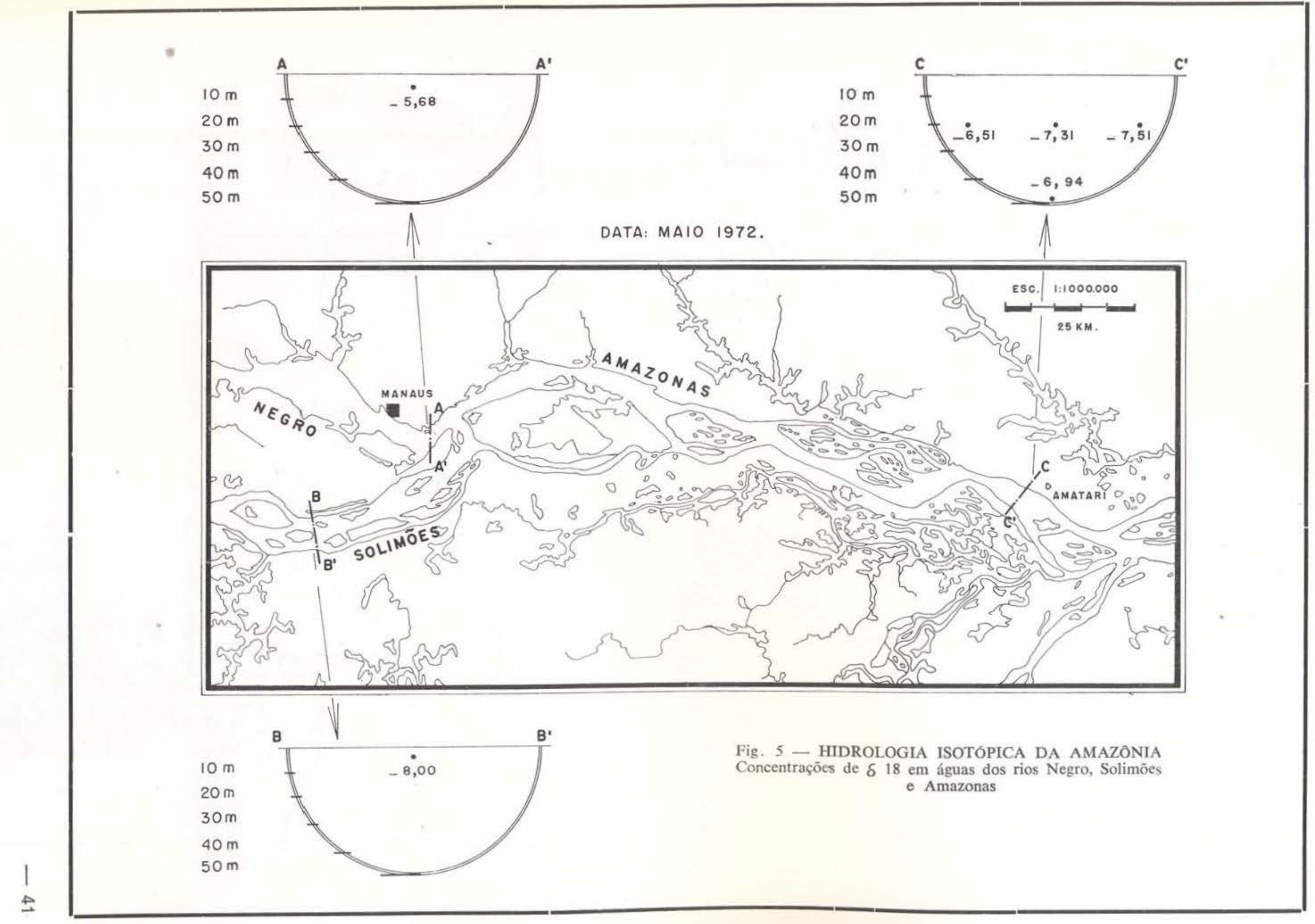




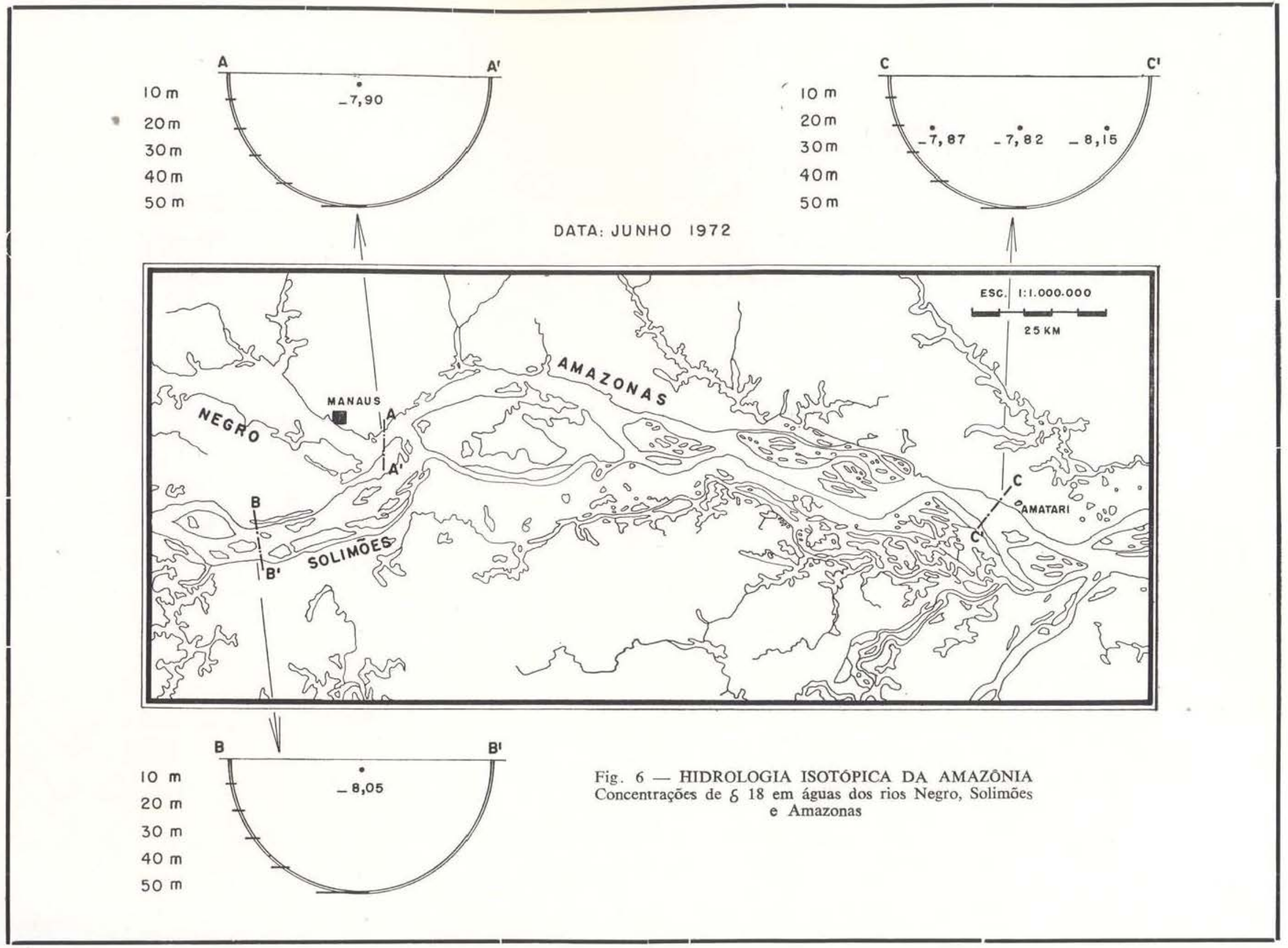




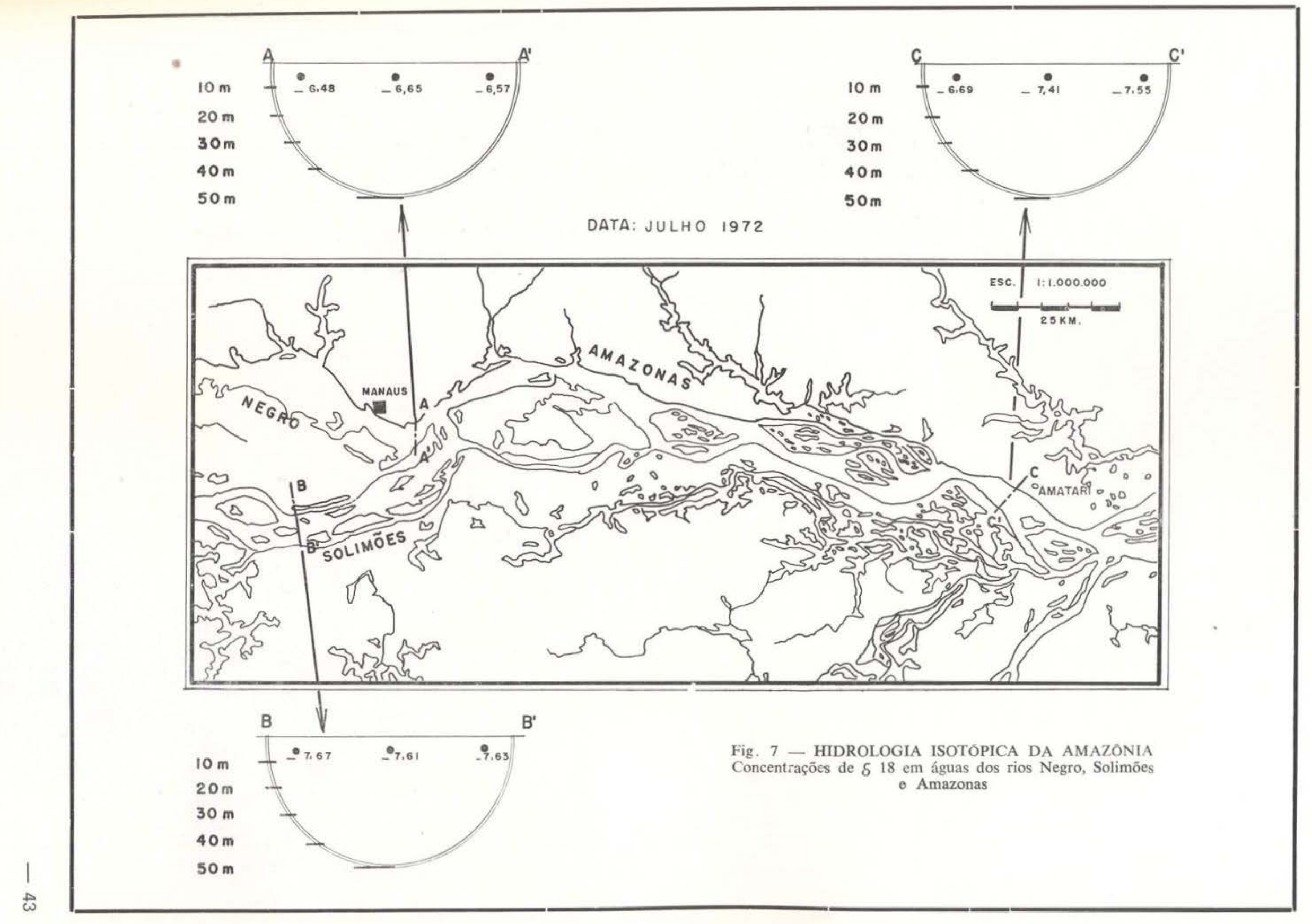




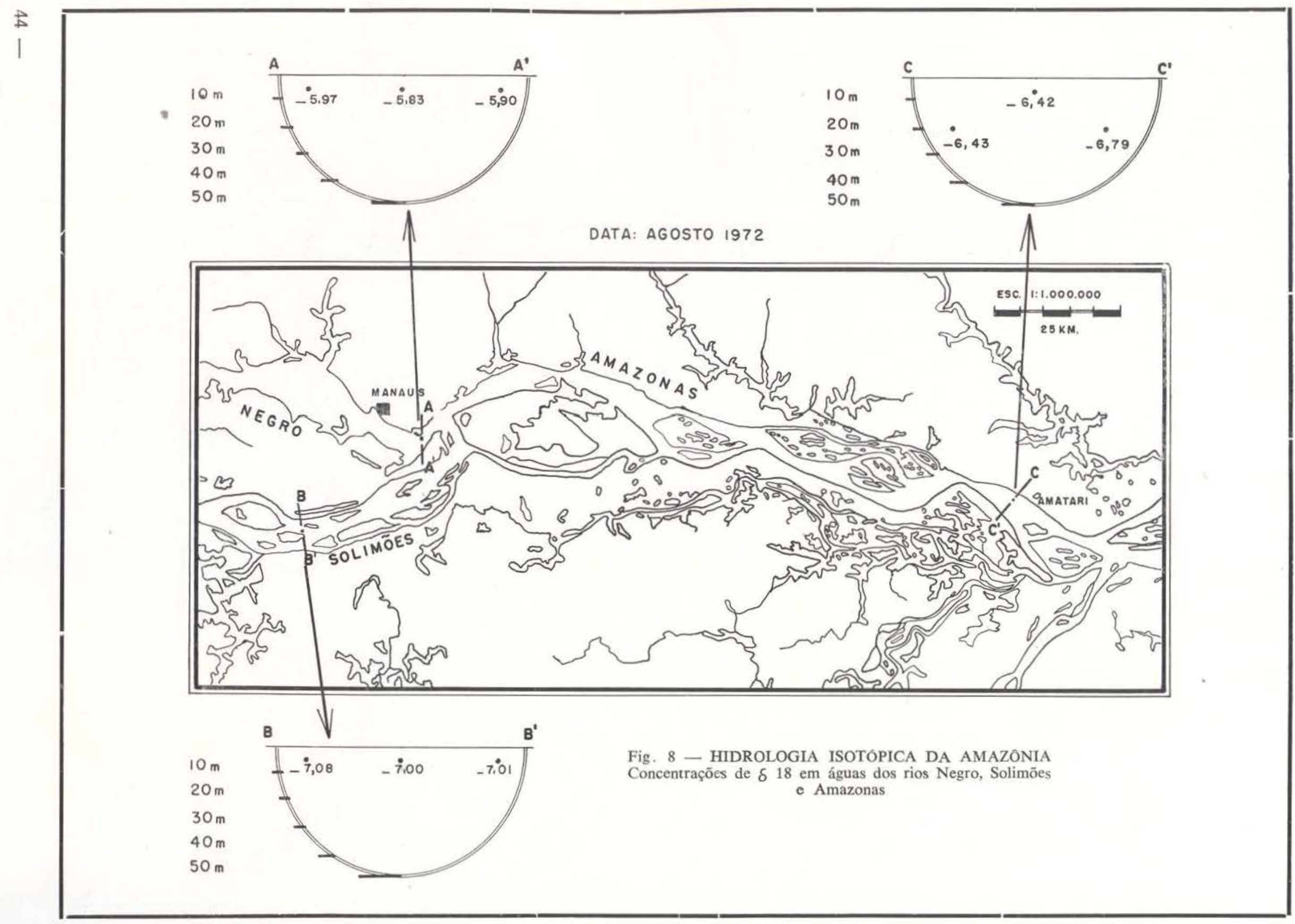




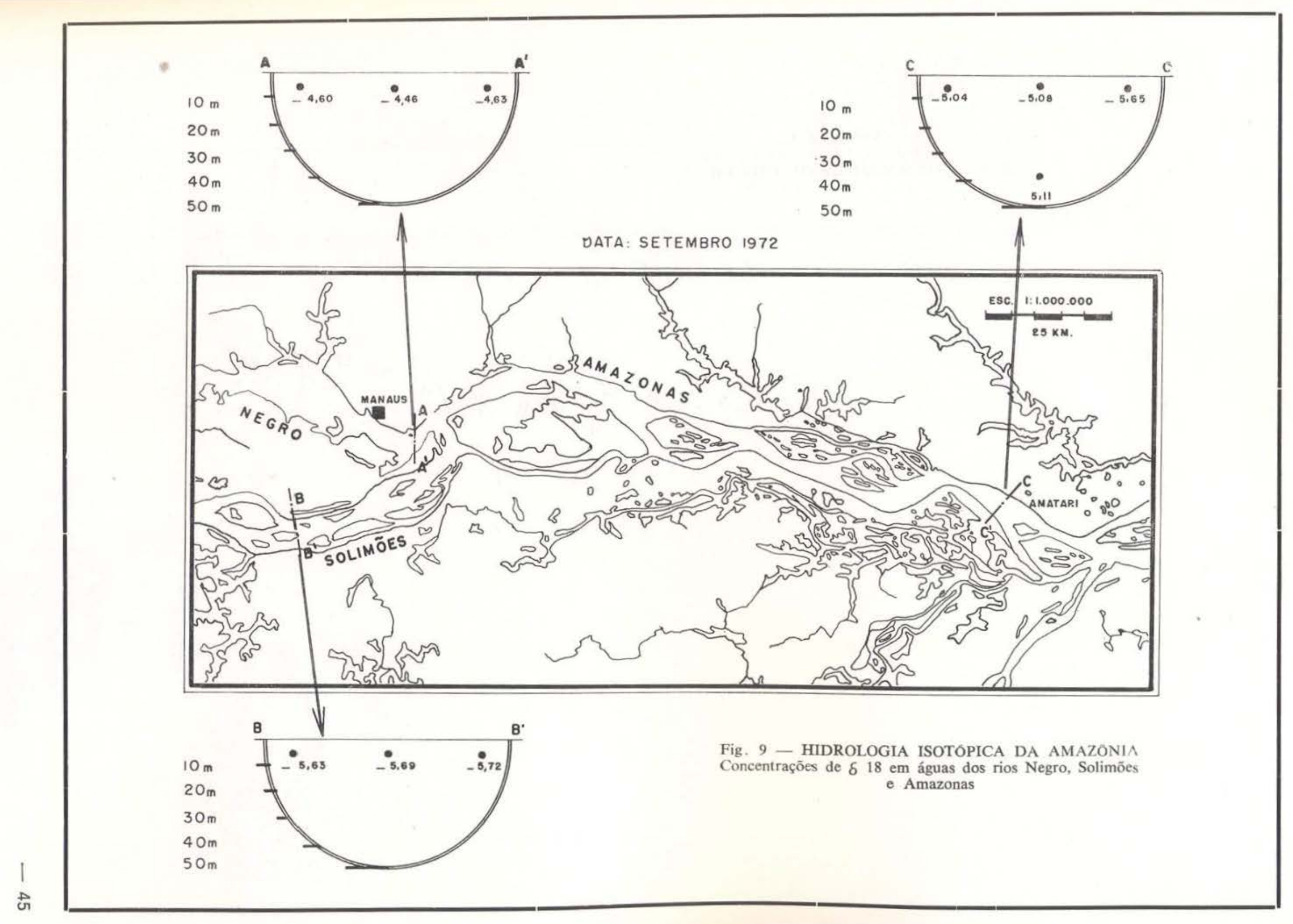




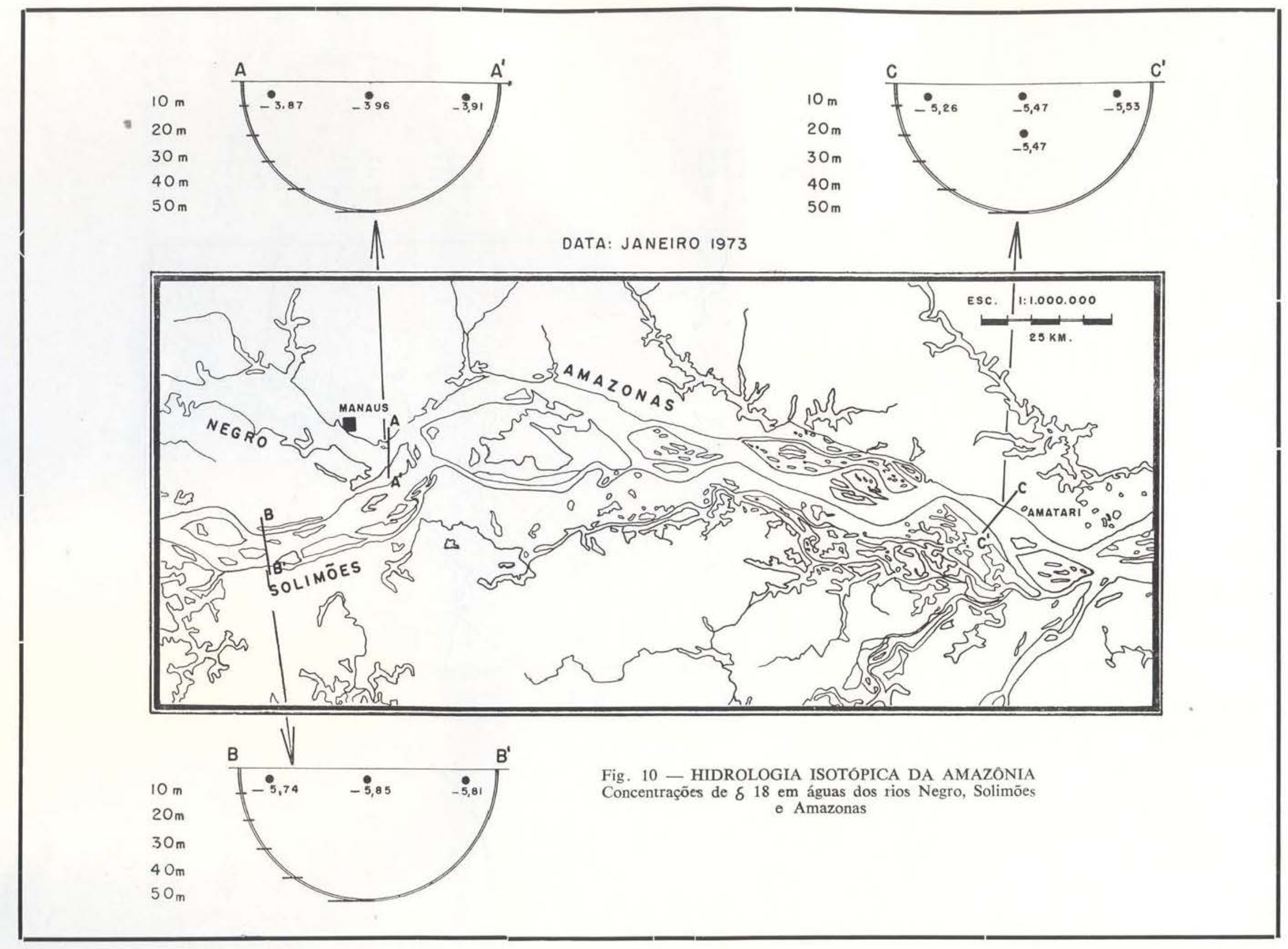

